\title{
Tanshinone IIA decreases the protein expression of EGFR, and IGFR blocking the PI3K/Akt/mTOR pathway in gastric carcinoma AGS cells both in vitro and in vivo
}

\author{
CHIN-CHENG SU ${ }^{1-4}$ and TSUNG-LANG CHIU ${ }^{5,6}$
}

\begin{abstract}
${ }^{1}$ Tumor Research Center of Integrative Medicine, ${ }^{2}$ Comprehensive Breast Cancer Center and ${ }^{3}$ Department of Surgery, Changhua Christian Hospital, Changhua 50006; ${ }^{4}$ School of Chinese Medicine, College of Chinese Medicine, China Medical University, Taichung 40402; ${ }^{5}$ Division of Neurooncology, Neuro-Medical Scientific Center, Buddhist Tzu-Chi General Hospital and ${ }^{6}$ Tzu Chi University, Hualien 97004, Taiwan, R.O.C.
\end{abstract}

Received January 31, 2016; Accepted April 19, 2016

DOI: $10.3892 /$ or.2016.4857

\begin{abstract}
Tan-IIA exerts powerful inhibitory effects in gastric cancer AGS cells. The PI3K/AKT/mTOR pathway is one of the most frequently dysregulated kinase cascades in human cancer. In the present study, we investigated the protein expression levels of PI3K, AKT and mTOR in AGS cells treated with Tan-IIA both in vitro and in vivo. The AGS cells were treated with Tan-IIA for different durations in vitro. In the in vivo study, AGS cell xerograft SCID mice were treated with Tan-IIA for 8 weeks. Subsequently, the protein expression of EGFR, IGFR, PI3K, AKT and mTOR was measured by western blotting. The results showed that Tan-IIA was able to decrease the protein expression levels of EGFR, IGFR, PI3K, AKT and mTOR significantly and dose-dependently in vitro and in vivo. In conclusion, these findings indicate Tan-IIA could inhibit AGS cells through decreasing the protein expression of EGFR, IGFR and blocking PI3K/AKT/mTOR pathway both in vitro and in vivo.
\end{abstract}

\section{Introduction}

According to the International Agency for Research on Cancer, the number of estimated new cases of gastric cancer in worldwide was approximately 989,600, and there were approximately 748,000 estimated deaths due to gastric cancer in the worldwide in 2008 (1). Tanshinone IIA (Tan-IIA; $\mathrm{C}^{19} \mathrm{H}^{18} \mathrm{O}^{3}$ ), is one of the diterpine quinones extracted from

Correspondence to: Dr Chin-Cheng Su, Tumor Research Center of Integrative Medicine, Changhua Christian Hospital, 135 Nan-Hsiao Street, Changhua 50006, Taiwan, R.O.C.

E-mail: succ.maeva@msa.hinet.net

Key words: Tanshinone IIA, PI3K/AKT/mTOR, gastric cancer, EGFR
Danshen (Salviae miltiorrhizae radix) (2,3), with anti-inflammatory activities $(4,5)$ and antioxidant properties $(6,7)$. It is well documented that Tanshinone IIA can inhibit many human cancer cell lines through different molecular mechanisms (8-15). PI3K can prevent apoptosis and promote cellular survival and proliferation in a wide variety of cells. Akt is a serine/threonine-specific protein kinase, which plays a key role in apoptosis and cell proliferation. Raha et al showed that Naringin induces autophagy-mediated growth inhibition by downregulating the PI3K/Akt/mTOR cascade via activation of MAPK pathways in AGS cancer cells (16). Lee et al also showed that flavonoids isolated from Citrus platymamma induce mitochondrial-dependent apoptosis in AGS cells by modulation of the PI3K/AKT and MAPK pathways (17). Zheng et al documented that Paeoniflorin inhibits human gastric carcinoma cell proliferation through suppression of PI3K/Akt and STAT3 signaling (18). These documents showed that agents can inhibit AGS cells through downregulating the PI3K/Akt/mTOR cascade. Tan-IIA could inhibit human gastric cancer SGC7901 cells and MKN-45 cells time- and dose-dependently through inducing apoptosis and cell cycle phase arrest (19-21). Tan-IIA also inhibited human gastric cancer AGS cells; one of the molecular mechanisms may be through MAPK pathways to induce G2/M phase arrest. The other may be through extrinsic apoptotic signaling pathway to induce apoptosis in vitro $(22,23)$. Tan-IIA shows potential as an alternative therapeutic agent for human gastric carcinoma. However, the underlying anticancer mechanism still needs to be explored further. In the present study, we investigated the protein expression levels of EGFR, IGFR, PI3K, AKT, mTOR and PTEN in human gastric cancer AGS cells treated with Tan-IIA in vitro and in vivo.

\section{Materials and methods}

The EGFR (\#2239, MW 175 kDa), IGFR (\#3018, MW 95 kDa), PI3K (\#4292, MW 85 kDa), AKT (\#3063, MW 60 kDa), mTOR (\#2983, MW 289 kDa) and PTEN (\#9559, MW 54 kDa) antibodies were all obtained from Cell Signaling Technology Inc. (Beverly,MA,USA).Tan-IIA was obtained from Sigma-Aldrich 
(St. Louis, MO, USA; CAS-No 568-72-9). Fetal bovine serum (FBS), F-12K medium, glutamine and penicillin-streptomycin were obtained from Gibco-BRL (Grand Island, NY, USA). Triton X-100, Tris- $\mathrm{HCl}$, ribonuclease-A, sodium deoxycholate, leupeptin, sodium pyruvate, HEPES, dimethyl sulfoxide (DMSO), 3-(4,5-dimethylthiazol-2-y1)-2,5-diphenyltetrazolium bromide (MTT) and Tween-20, mouse anti- $\beta$-actin were obtained from Sigma-Aldrich. Potassium phosphate and $0.2 \mathrm{~mm}$ PVFD membranes were purchased from Merck Co. (Darmstadt, Germany); the AGS human gastric adenocarcinoma cell line (BCRC number: 60102) was obtained from the Food Industry Research and Development Institute (Hsinchu, Taiwan). BioMax film was obtained from Kodak.

Male SCID mice. Sixty male SCID mice, weighing 10-15 g (3-4 weeks old), were obtained from the Laboratory Animal Center, Tzu Chi University (Hualien, Taiwan).

Cell culture. The human gastric adenocarcinoma AGS cells were obtained from the Food Industry Research and Development Institute (Hsinchu, Taiwan). The cell culture procedure was as described $(22,23)$. Briefly, the AGS cells were placed into $75-\mathrm{cm}^{2}$ tissue culture flasks and maintained in F-12K contained with $10 \%$ heat-inactivated FBS (Gibco-BRL), $100 \mathrm{U} / \mathrm{ml}$ penicillin and $100 \mu \mathrm{g} / \mathrm{ml}$ streptomycin. Cells were grown at $37^{\circ} \mathrm{C}$ in a humidified atmosphere of $95 \%$ air and $5 \% \mathrm{CO}_{2}$.

The protein expression of EGFR, IGFR, PI3K, AKT, mTOR, $P T E N$ and $\beta$-actin in AGS cells treated with various concentrations of Tan-IIA. The AGS cells were treated with various concentrations of Tan-IIA $(0,2.0,3.7$ and $5.5 \mu \mathrm{g} / \mathrm{ml})$ for 24 or $48 \mathrm{~h}$ and then the protein expression levels of EGFR, IGFR, PI3K, AKT, mTOR, PTEN and $\beta$-actin were evaluated by western blot analysis.

The protein expressions of EGFR, IGFR, PIJK, AKT, mTOR, $P T E N$ and $\beta$-actin in AGS cells treated with Tan-IIA for different durations. The AGS cells were treated with Tan-IIA $(3.7 \mu \mathrm{g} / \mathrm{ml})$ for different durations $(0,24$ and $48 \mathrm{~h})$ and then the protein expression levels of VEGFR, HER2, Ras, Raf, MEK, ERK, PARP, caspase- 3 and $\beta$-actin were evaluated by western blot analysis.

Effects of Tan-IIA on the protein expression of EGFR, IGFR, $P I 3 K, A K T$, mTOR, PTEN and $\beta$-actin in AGS cell xenograft tumors. Three-week-old male nude SCID mice (number $=60$ ) were xenograft with AGS cells $\left(2 \times 10^{6} / 0.2 \mathrm{ml}\right)$ and maintained in a pathogen-free environment (CCH-AE-102-007; Laboratory Animal Center of Tzu Chi University, Hualien, Taiwan). When the xenograft tumors reached more than $0.5 \mathrm{~cm}$ in diameter, the mice were divided randomly into four groups. Tan-IIA was dissolved in corn oil and then administered to the mice at concentrations of 30,60 and $90 \mathrm{mg} / \mathrm{kg}$, QW1, 3, 5 by intraperitoneum injection for 8 weeks. The control group was treated with an equal volume of corn oil. SCID mice were scarified by $\mathrm{CO}_{2}$ inhalation and then the xenograft tumors were dissected. All animal experiments were approved by the Institutional Animal Care and Use Committee (IACUC), approval no. CCH-AE-102-007). Subsequently, the protein expressions of EGFR, IGFR, PI3K, AKT, mTOR and PTEN in the tumors were measured by western blotting.
Protein preparation. Proteins were extracted from xenograft tumors. The xenograft tumors were dissected and ground, then the thick liquid $(0.06 \mathrm{gm})$ was lysed in the ice-cold whole cell extract buffer containing the protease inhibitors. The lysate was vibrated for $30 \mathrm{~min}$ at $4^{\circ} \mathrm{C}$ and centrifuged at $12,281 \mathrm{x} \mathrm{g}$ for $10 \mathrm{~min}$. Protein concentration was measured by BCA protein assay kit (Pierce, Rockford, IL, USA).

Western blot analysis. The western blot procedures were as described $(22,23)$. Briefly, AGS cells were treated with various concentrations of Tan-IIA for different durations, and then the cells were lysed in the ice-cold whole cell extract buffer containing the protease inhibitors. The lysate was vibrated for $30 \mathrm{~min}$ at $4^{\circ} \mathrm{C}$ and centrifuged at $12,281 \mathrm{x} \mathrm{g}$ for $10 \mathrm{~min}$. Protein concentration was measured by BCA protein assay kit (Pierce). Equal amounts of proteins were subjected to electrophoresis using $12 \%$ sodium dodecyl sulfate-polyacrylamide gels. To verify equal protein loading and transfer, proteins were then transferred to polyvinylidene difluoride membranes and the membranes were blocked for $1 \mathrm{~h}$ at $4^{\circ} \mathrm{C}$ using blocking buffer [5\% non-fat dried milk in solution containing $50 \mathrm{mM}$ Tris/ $\mathrm{HCl}$ ( $\mathrm{pH} \mathrm{8.0),} 2 \mathrm{mM} \mathrm{CaCl}_{2}, 80 \mathrm{mM}$ sodium chloride, $0.05 \%$ Tween- 20 and $0.02 \%$ sodium azide]. The membranes were then incubated for $2 \mathrm{~h}$ at room temperature with specific primary antibody followed by anti-rabbit or anti-mouse immunoglobulin G-horseradish peroxidase conjugated secondary antibodies. The membranes were washed three times for 10 min with washing solution. Finally, the protein bands were visualized on the X-ray film using the enhanced chemiluminescence detection system (PerkinElmer Life and Analytical Sciences, Boston, MA, USA).

Statistical analysis. Values are presented as the means \pm SD. The Student's t-test was used to analyze statistical significance. A p-value of $<0.05$, was considered to indicate a statistically significant difference for all the tests. $\mathrm{P}<0.05, \mathrm{P}<0.01, \mathrm{P}<0.001$.

\section{Results}

Effects of Tan-IIA on the viability of AGS cells. The results revealed that Tan-IIA can inhibit AGS cells in a time- and dose-dependent manner. The half-maximal inhibitory concentration $\left(\mathrm{IC}_{50}\right)$ was $5.5,3.7$ and $3.5 \mu \mathrm{g} / \mathrm{ml}$ at 24,48 and $72 \mathrm{~h}$, respectively (data not show). This is agreement with our previous studies $(22,23)$.

Effects of Tan-IIA on the protein expression of EGFR, IGFR, PI3K, AKT, mTOR, PTEN and $\beta$-actin in AGS cells. The AGS cells were treated with various concentrations of Tan-IIA $(0$, 2.0, 3.7 and $5.5 \mu \mathrm{g} / \mathrm{ml}$ ) for 24 or $48 \mathrm{~h}$ and then the protein expression levels of EGFR, IGFR, PI3K, AKT, mTOR, p-TEN and $\beta$-actin were evaluated by western blot analysis. The results showed that Tan-IIA can decrease the protein expression levels of EGFR (Fig. 1A), IGFR (Fig. 1B), PI3K (Fig. 1C), AKT (Fig. 1D), mTOR (Fig. 1E) and PTEN (Fig. 1F) significantly.

Effects of Tan-IIA on the protein expression of EGFR, IGFR, PI3K, AKT, mTOR, PTEN and $\beta$-actin in AGS cells. The AGS cells were treated with Tan-IIA $(3.7 \mu \mathrm{g} / \mathrm{ml})$ for different 
A
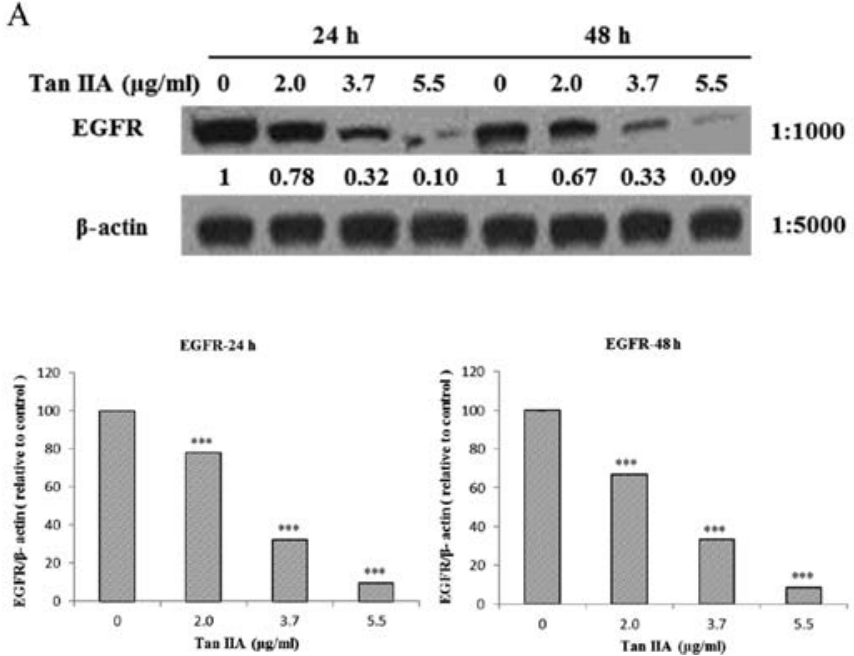

C
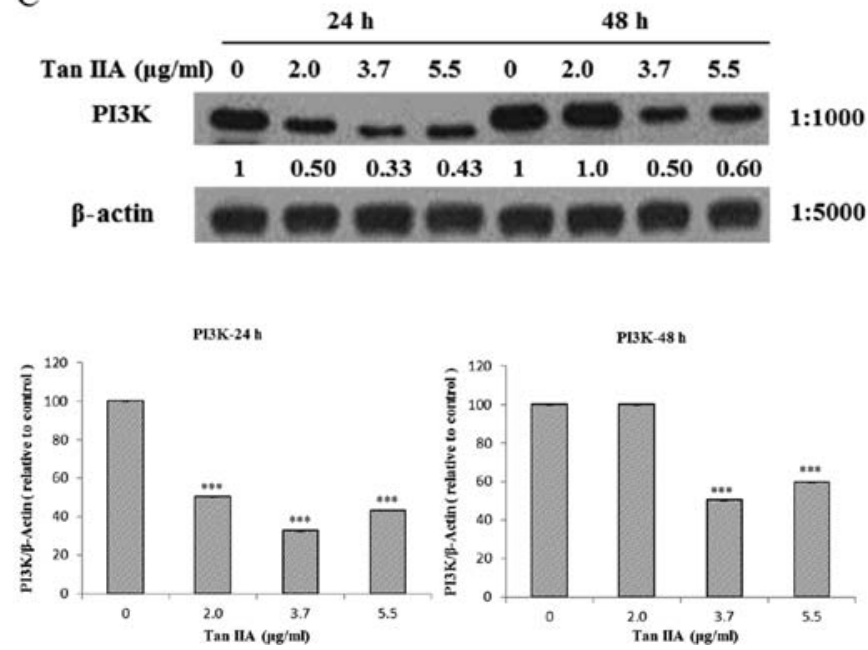

E
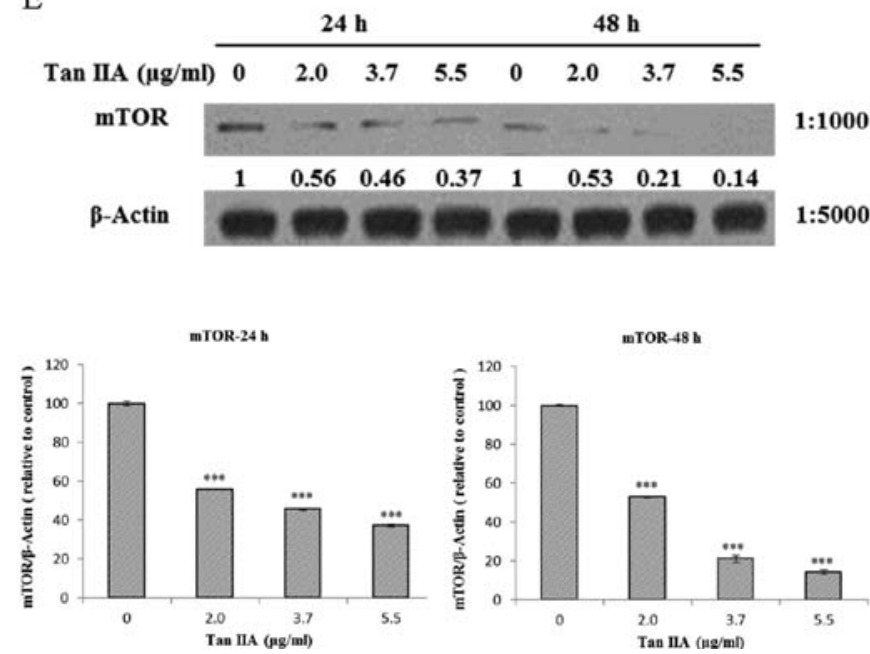

B

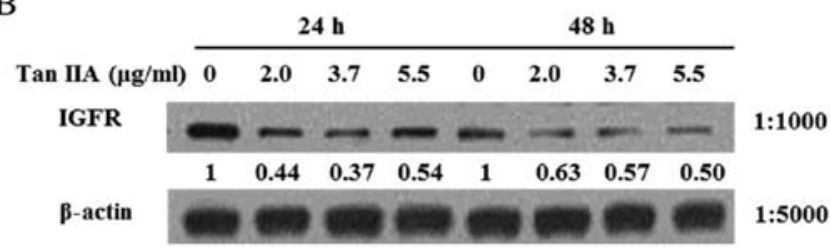

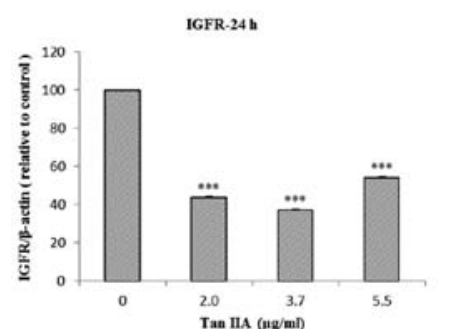

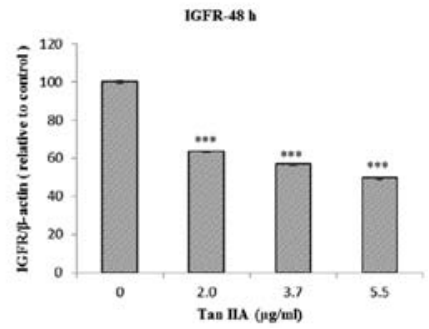

D
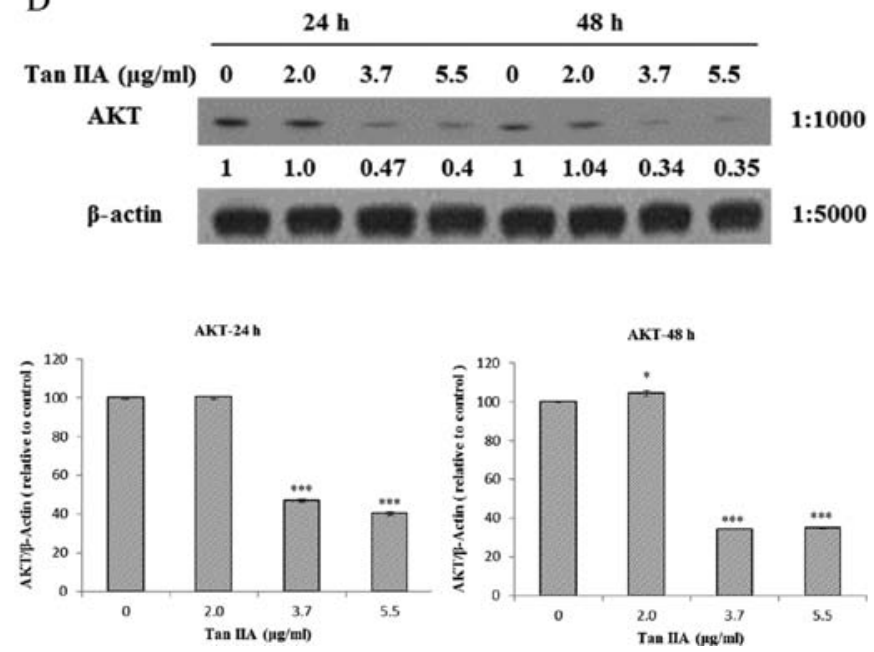

F
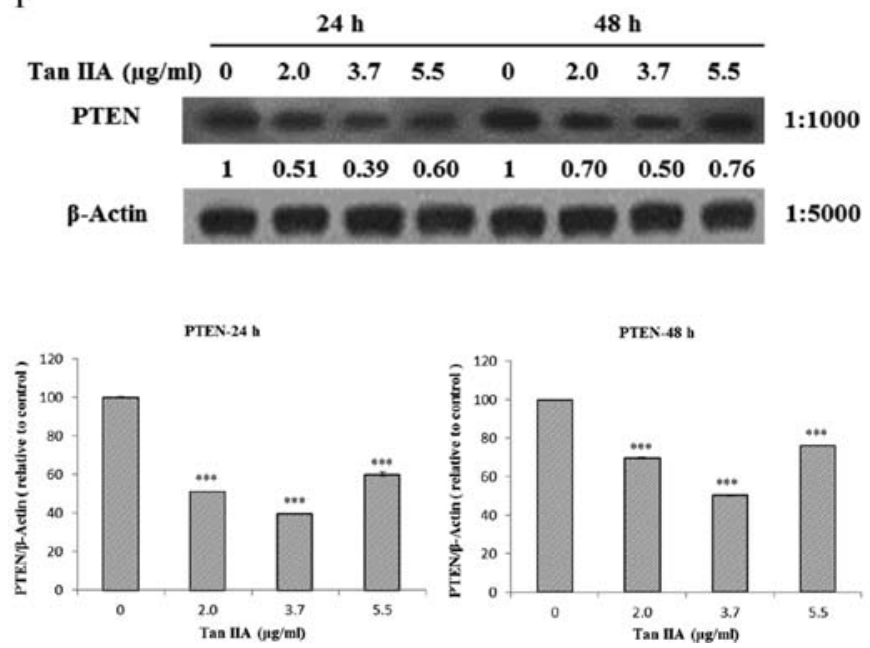

Figure 1. Effects of Tan-IIA on the protein expression of EGFR, IGFR, PI3K, AKT, mTOR, p-TEN and $\beta$-actin in AGS cells. The AGS cells were treated with various concentrations of Tan-IIA $(0,2.0,3.7$ and $5.5 \mu \mathrm{g} / \mathrm{ml})$ for 24 or $48 \mathrm{~h}$ and then the protein expression levels were evaluated by western blot analysis as described in Materials and methods. The results showed that Tan-IIA can decrease the protein expression levels of EGFR (A), IGFR (B), PI3K (C), AKT (D), mTOR (E) and p-TEN (F) significantly and dose-dependently. ${ }^{*} \mathrm{P}<0.05$, was considered to indicate a statistically significant difference for all the tests. ${ }^{*} \mathrm{P}<0.05$, ${ }^{* *} \mathrm{P}<0.01,{ }^{* * *} \mathrm{P}<0.001$.

durations $(0,24$ and $48 \mathrm{~h})$ and then the protein expression levels of EGFR, IGFR, PI3K, AKT, mTOR, p-TEN and $\beta$-actin were evaluated by western blot analysis. The results showed that Tan-IIA can decrease the protein expression 
A
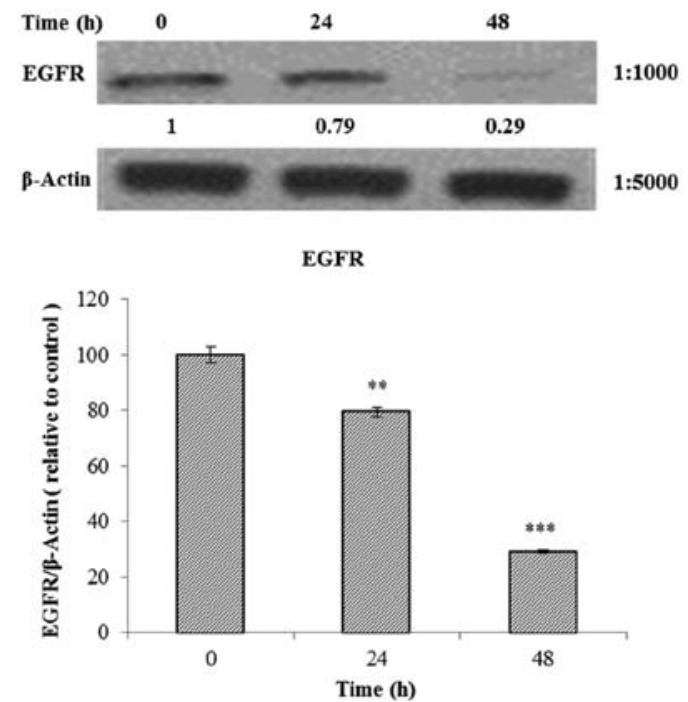

$\mathrm{C}$

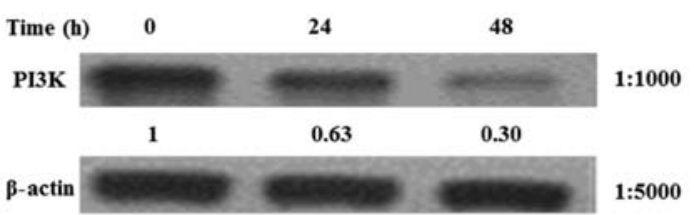

PI3K

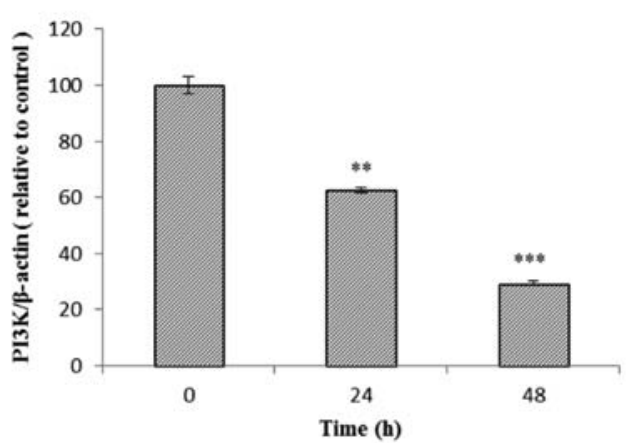

E

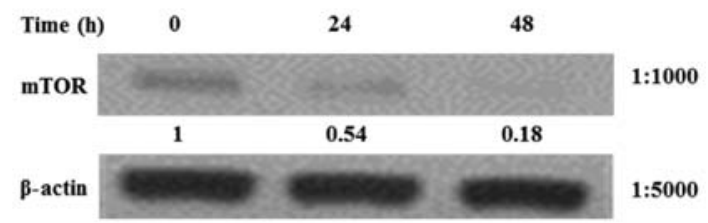

mTOR

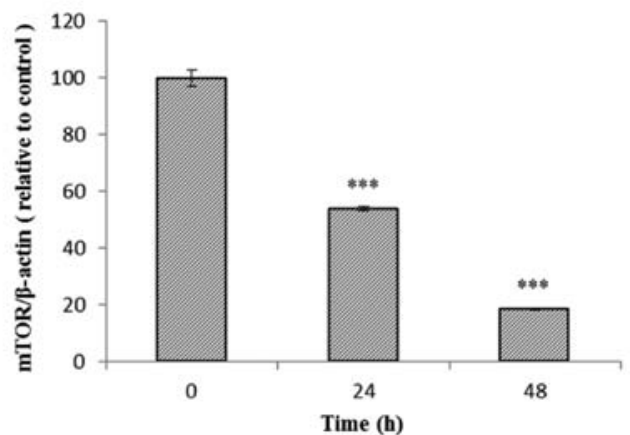

B

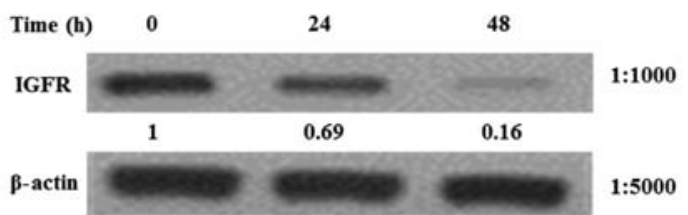

IGFR

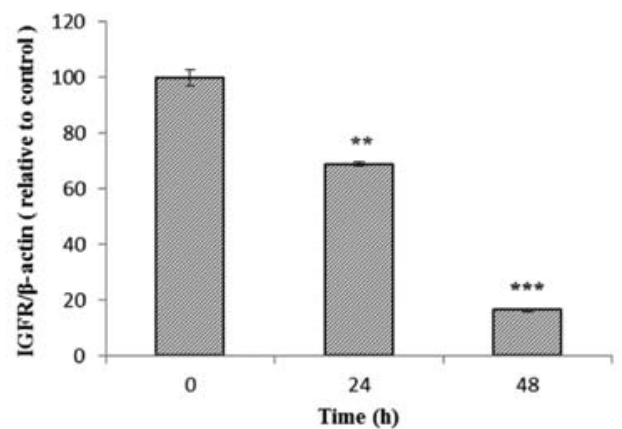

$\mathrm{D}$

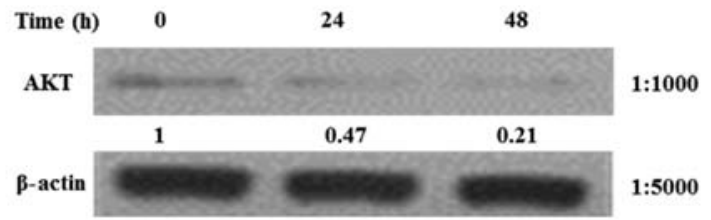

AKT
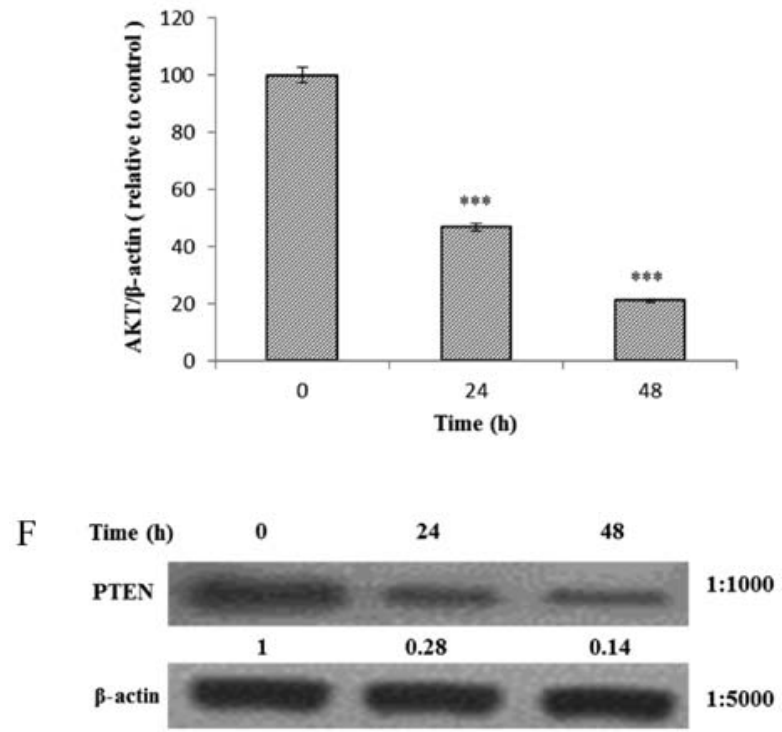

PTEN

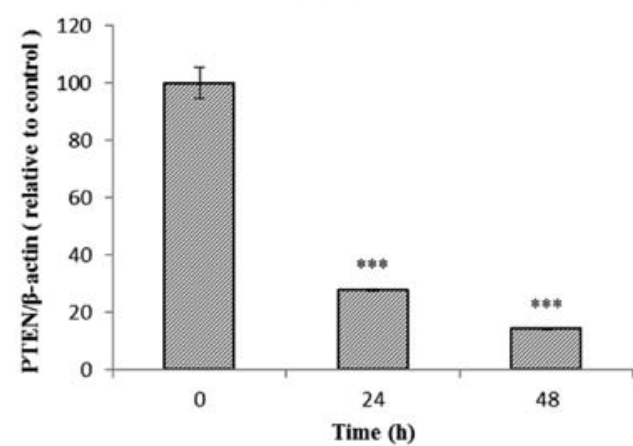

Figure 2. Effects of Tan-IIA on the protein expression of EGFR, IGFR, PI3K, AKT, mTOR, p-TEN and $\beta$-actin in AGS cells. The AGS cells were treated with Tan-IIA $(3.7 \mu \mathrm{g} / \mathrm{ml})$ for different durations $(0,24$ and $48 \mathrm{~h})$ and then the protein expression levels were evaluated by western blot analysis as described in Materials and methods. The results showed that Tan-IIA can decrease the protein expression levels of EGFR (A), IGFR (B), PI3K (C), AKT (D), mTOR (E) and p-TEN (F) significantly and dose-dependently. ${ }^{*} \mathrm{P}<0.05$ was considered to indicate a statistically significant difference for all the tests. ${ }^{*} \mathrm{P}<0.05,{ }^{* *} \mathrm{P}<0.01$, ${ }^{* * *} \mathrm{P}<0.001$. 

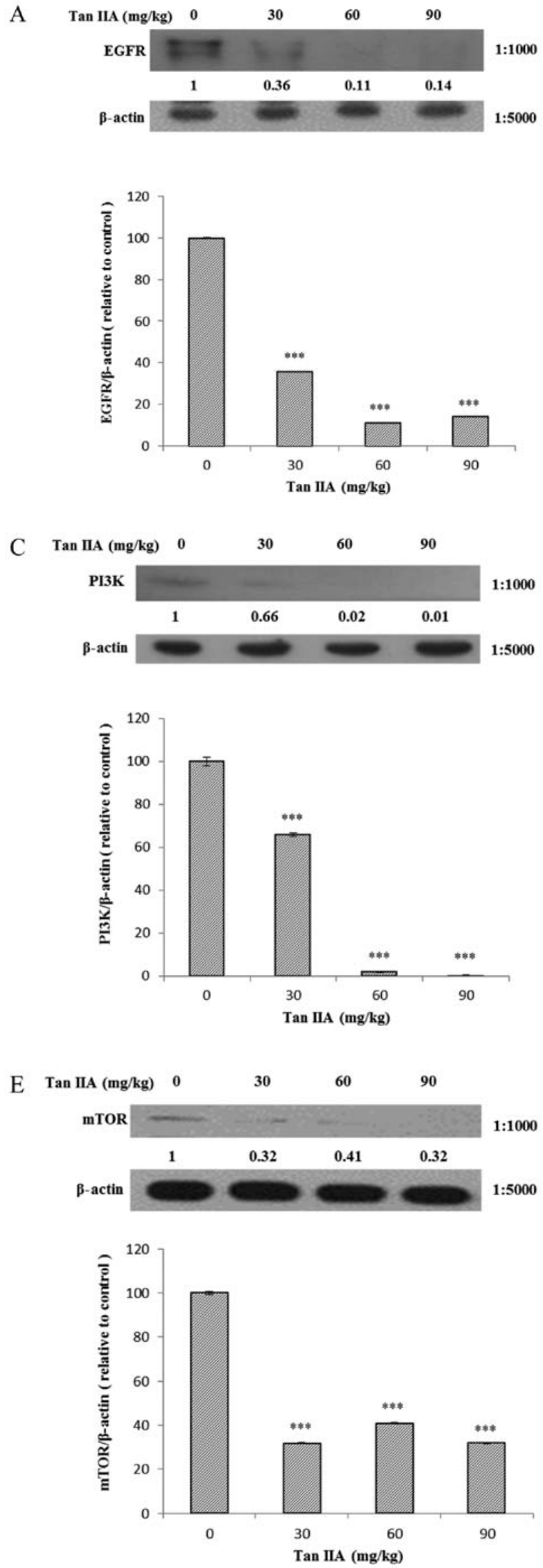
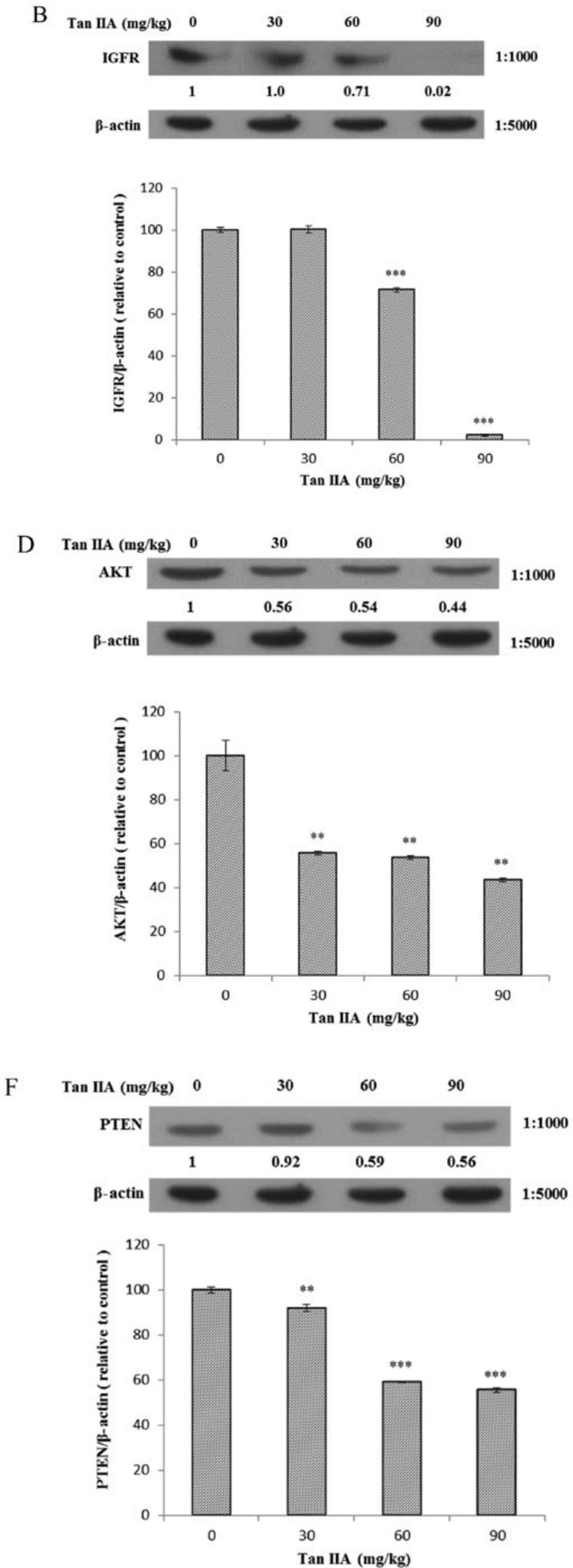

Figure 3. Effects of Tan-IIA on the protein expressions of EGFR, IGFR, PI3K, AKT, mTOR, p-TEN and $\beta$-actin in AGS cell xenograft tumors. AGS cell xenograft tumors were treated with different doses of Tan-IIA then the protein expressions were measured by western blotting as described in Materials and methods. The results showed that Tan-IIA can decrease the protein expression levels of EGFR (A), IGFR (B), PI3K (C), AKT (D), mTOR (E) and p-TEN (F) significantly and dose-dependently. ${ }^{*} \mathrm{P}<0.05$ was considered to indicate a statistically significant difference for all the tests. ${ }^{*} \mathrm{P}<0.05,{ }^{* *} \mathrm{P}<0.01,{ }^{* * * *} \mathrm{P}<0.001$. 


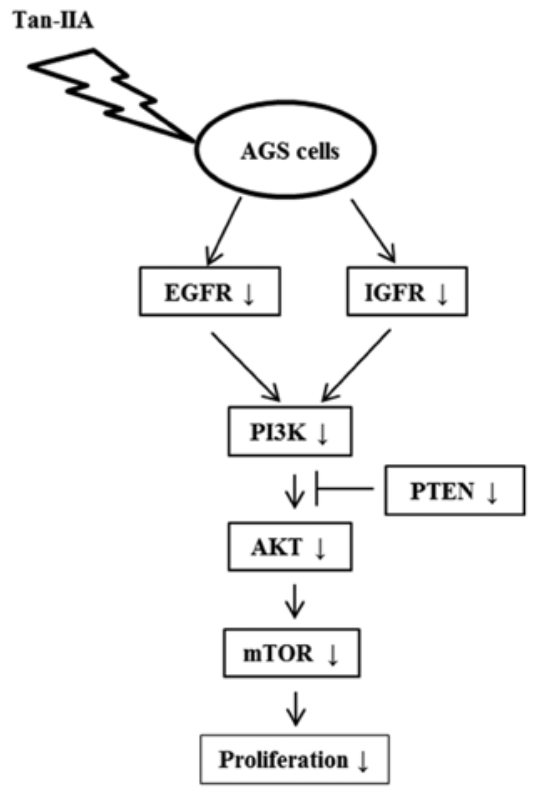

Figure 4. The proposed model for Tan-IIA to inhibit the proliferation of AGS cells. Tan-IIA inhibits human gastric cancer AGS cells through decreasing the protein expression of EGFR, IGFR and blocking PI3K/AKT/mTOR pathway.

levels of EGFR (Fig. 2A), IGFR (Fig. 2B), PI3K (Fig. 2C), AKT (Fig. 2D), mTOR (Fig. 2E) and PTEN (Fig. 2F) significantly.

Effects of Tan-IIA on the protein expression of EGFR, IGFR, PI3K, AKT, mTOR, PTEN and $\beta$-actin in AGS cell xenograft tumors. The AGS cell xenograft tumor SCID mice were treated with different doses of Tan-IIA for 8 weeks then sacrificed. The protein expression of EGFR, IGFR, PI3K, AKT, mTOR and PTEN in xenograft tumors were measured by western blotting as described in Materials and methods. The results showed that Tan-IIA can decrease the protein expression levels of EGFR (Fig. 3A), IGFR (Fig. 3B), PI3K (Fig. 3C), AKT (Fig. 3D), mTOR (Fig. 3E) and PTEN (Fig. 3F) significantly and dose-dependently.

\section{Discussion}

It is well documented that PI3K/AKT/mTOR pathway is one of the most frequently dysregulated kinase cascades in human cancer $(24,25)$. PI3K/PTEN/Akt/mTOR cascade inhibitors have been investigated in pre-clinical and clinical investigations and reported as having potential (26). The transmembrane tyrosine kinases, such as insulin-like growth factor receptor (IGFR) or epidermal growth factor receptor (EGFR) have been strongly implicated in the growth, survival, and metastasis of a wide variety of human tumors $(27,28)$. PI3K/PTEN/Akt/mTOR pathway represents important signal transduction mechanisms that facilitate the proliferation and survival of cancers driven by growth factor receptors (29).

It has been well documented that Naringin, flavonoids and Paeoniflorin can inhibit AGS cells through downregulating the PI3K/Akt/mTOR cascade (16-18). Our results showed that
AGS cells treated with Tan-IIA can downregulate the protein expressions of PI3K/ Akt/mTOR both in vitro and in vivo.

Protein phosphatase activity is closely associated with tumors. PI3K is necessary for the activation of AKT. PTEN regulates the activity of AKT via activated phosphatidylinositol triphosphate (PIP3) (30). Our results showed that AGS cells treated with Tan-IIA can downregulate the protein expression of PTEN both in vitro and in vivo. These findings indicate Tan-IIA can inhibit the proliferation of AGS cells, one of the molecular mechanisms may be through downregulating the $\mathrm{PI} 3 \mathrm{~K} / \mathrm{Akt} / \mathrm{mTOR}$ cascade. The proposed model is shown in Fig. 4. This is the first report that Tan-IIA could inhibit gastric carcinoma AGS cells by decreasing the protein expression of EGFR, IGFR and blocking the PI3K/Akt/mTOR pathway in a cancer-bearing animal model.

\section{Acknowledgements}

This study was supported by grant 103-CCH-IRP-023 from Changhua Christian Hospital from the Research Section of the Changhua Christian Hospital (Changhua, Taiwan).

\section{References}

1. Jemal A, Bray F, Center MM, Ferlay J, Ward E and Forman D: Global cancer statistics. CA Cancer J Clin 61: 69-90, 2011.

2. Che AJ, Zhang JY, Li CH, Chen XF, Hu ZD and Chen XG: Separation and determination of active components in Radix Salviae miltiorrhizae and its medicinal preparations by nonaqueous capillary electrophoresis. J Sep Sci 27: 569-575, 2004.

3. Zhou L, Zuo Z and Chow MS: Danshen: An overview of its chemistry, pharmacology, pharmacokinetics, and clinical use. J Clin Pharmacol 45: 1345-1359, 2005.

4. Jang SI, Kim HJ, Kim YJ, Jeong SI and You YO: Tanshinone IIA inhibits LPS-induced NF-kappaB activation in RAW 264.7 cells: Possible involvement of the NIK-IKK, ERK1/2, p38 and JNK pathways. Eur J Pharmacol 542: 1-7, 2006.

5. Li W, Li J, Ashok M, Wu R, Chen D, Yang L, Yang H, Tracey KJ, Wang P, Sama AE, et al: A cardiovascular drug rescues mice from lethal sepsis by selectively attenuating a late-acting proinflammatory mediator, high mobility group box 1 . J Immunol 178: 3856-3864, 2007.

6. Wang AM, Sha SH, Lesniak W and Schacht J: Tanshinone (Salviae miltiorrhizae extract) preparations attenuate aminoglycoside-induced free radical formation in vitro and ototoxicity in vivo. Antimicrob Agents Chemother 47: 1836-1841, 2003.

7. Lin R, Wang WR, Liu JT, Yang GD and Han CJ: Protective effect of tanshinone IIA on human umbilical vein endothelial cell injured by hydrogen peroxide and its mechanism. J Ethnopharmacol 108: 217-222, 2006.

8. Su CC and Lin YH: Tanshinone IIA down-regulates the protein expression of ErbB-2 and up-regulates TNF- $\alpha$ in colon cancer cells in vitro and in vivo. Int J Mol Med 22: 847-851, 2008.

9. Su CC and Lin YH: Tanshinone IIA inhibits human breast cancer cells through increased Bax to Bcl-xL ratios. Int J Mol Med 22: 357-361, 2008.

10. Chiu TL and Su CC: Tanshinone IIA induces apoptosis in human lung cancer A549 cells through the induction of reactive oxygen species and decreasing the mitochondrial membrane potential. Int J Mol Med 25: 231-236, 2010.

11. Cheng CY and Su CC: Tanshinone IIA may inhibit the growth of small cell lung cancer $\mathrm{H} 146$ cells by up-regulating the Bax/Bcl-2 ratio and decreasing mitochondrial membrane potential. Mol Med Rep 3: 645-650, 2010.

12. Cheng CY and Su CC: Tanshinone IIA inhibits Hep-J5 cells by increasing calreticulin, caspase 12 and GADD153 protein expression. Int J Mol Med 26: 379-385, 2010.

13. Yan MY, Chien SY, Kuo SJ, Chen DR and Su CC: Tanshinone IIA inhibits BT-20 human breast cancer cell proliferation through increasing caspase 12, GADD153 and phospho-p38 protein expression. Int J Mol Med 29: 855-863, 2012. 
14. Huang CY, Chiu TL, Kuo SJ, Chien SY, Chen DR and Su CC: Tanshinone IIA inhibits the growth of pancreatic cancer BxPC 3 cells by decreasing protein expression of TCTP, MCL 1 and Bcl xL. Mol Med Rep 7: 1045-1049, 2013.

15. Su CC: Tanshinone IIA could inhibit pancreatic cancer BxPC-3 cells through increasing PERK, ATF6, caspase-12 and CHOP expression to induce apoptosis. J BiSE 8: 149-159, 2015.

16. Raha S, Yumnam S, Hong GE, Lee HJ, Saralamma VV, Park HS, Heo JD, Lee SJ, Kim EH, Kim JA, et al: Naringin induces autophagy-mediated growth inhibition by downregulating the $\mathrm{PI} 3 \mathrm{~K} / \mathrm{Akt} / \mathrm{mTOR}$ cascade via activation of MAPK pathways in AGS cancer cells. Int J Oncol 47: 1061-1069, 2015.

17. Lee HJ, Nagappan A, Park HS, Hong GE, Yumnam S, Raha S, Saralamma VV,Lee WS, KimEH and Kim GS: Flavonoids isolated from Citrus platymamma induce mitochondrial-dependent apoptosis in AGS cells by modulation of the PI3K/AKT and MAPK pathways. Oncol Rep 34: 1517-1525, 2015.

18. Zheng YB, Xiao GC, Tong SL, Ding Y, Wang QS, Li SB and Hao ZN: Paeoniflorin inhibits human gastric carcinoma cell proliferation through up-regulation of microRNA-124 and suppression of PI3K/Akt and STAT3 signaling. World J Gastroenterol 21: 7197-7207, 2015.

19. Hou J, He J, Jin X, Hu T and Zhang Y: Study on optimisation of extraction process of tanshinone IIA and its mechanism of induction of gastric cancer SGC7901 cell apoptosis. Afr J Tradit Complement Altern Med 10: 456-458, 2013

20. Xu M, Cao FL, Li NY, Liu YQ, Li YP and Lv CL: Tanshinone IIA reverses the malignant phenotype of SGC7901 gastric cancer cells. Asian Pac J Cancer Prev 14: 173-177, 2013.

21. Dong X, Dong J, Peng G, Hou X and Wu G: Growth-inhibiting and apoptosis-inducing effects of Tanshinone II A on human gastric carcinoma cells. J Huazhong Univ Sci Technolog Med Sci 27: 706-709, 2007

22. Su CC: Tanshinone IIA inhibits human gastric carcinoma AGS cell growth by decreasing BiP, TCTP, Mcl 1 and $\mathrm{Bcl} x \mathrm{~L}$ and increasing Bax and CHOP protein expression. Int J Mol Med 34: $1661-1668,2014$
23. Su CC: Tanshinone IIA inhibits gastric carcinoma AGS cells through increasing p-p38, p-JNK and p53 but reducing p-ERK, CDC2 and cyclin B1 expression. Anticancer Res 34: 7097-7110, 2014.

24. Santarpia L, Lippman SM and El-Naggar AK: Targeting the MAPK-RAS-RAF signaling pathway in cancer therapy. Expert Opin Ther Targets 16: 103-119, 2012.

25. Liu P, Cheng H, Roberts TM and Zhao JJ: Targeting the phosphoinositide 3-kinase pathway in cancer. Nat Rev Drug Discov 8: 627-644, 2009.

26. McCubrey JA, Steelman LS, Chappell WH, Abrams SL, Franklin RA, Montalto G, Cervello M, Libra M, Candido S, Malaponte G, et al: Ras/Raf/MEK/ERK and PI3K/PTEN/Akt/mTOR cascade inhibitors: How mutations can result in therapy resistance and how to overcome resistance. Oncotarget 3: 1068-1111, 2012.

27. Yuen JS and Macaulay VM: Targeting the type 1 insulin-like growth factor receptor as a treatment for cancer. Expert Opin Ther Targets 12: 589-603, 2008.

28. Chitnis MM, Yuen JS, Protheroe AS, Pollak M and Macaulay VM: The type 1 insulin-like growth factor receptor pathway. Clin Cancer Res 14: 6364-6370, 2008.

29. McCubrey JA, Steelman LS, Kempf CR, Chappell WH, Abrams SL, Stivala F, Malaponte G, Nicoletti F, Libra M, Bäsecke J, et al: Therapeutic resistance resulting from mutations in Raf/MEK/ERK and PI3K/PTEN/Akt/mTOR signaling pathways. J Cell Physiol 226: 2762-2781, 2011.

30. Munroe ME, Businga TR, Kline JN and Bishop GA Anti-inflammatory effects of the neurotransmitter agonist Honokiol in a mouse model of allergic asthma. J Immunol 185: 5586-5597, 2010 